\title{
A Stochastic SIVS Epidemic Model Based on Birth and Death Process
}

\author{
Lin Zhu, Tiansi Zhang* \\ College of Science, University of Shanghai for Science and Technology, Shanghai, China \\ Email: *zhangts1209@163.com
}

How to cite this paper: Zhu, L. and Zhang, T.S. (2016) A Stochastic SIVS Epidemic Model Based on Birth and Death Process. Journai of Applied Mathematics and Physics, 4, 18371848.

http://dx.doi.org/10.4236/jamp.2016.49186

Received: August 13, 2016

Accepted: September 26, 2016

Published: September 29, 2016

Copyright $\odot 2016$ by authors and Scientific Research Publishing Inc. This work is licensed under the Creative Commons Attribution International License (CC BY 4.0).

http://creativecommons.org/licenses/by/4.0/

\begin{abstract}
A new stochastic epidemic model, that is, a general continuous time birth and death chain model, is formulated based on a deterministic model including vaccination. We use continuous time Markov chain to construct the birth and death process. Through the Kolmogorov forward equation and the theory of moment generating function, the corresponding population expectations are studied. The theoretical result of the stochastic model and deterministic version is also given. Finally, numerical simulations are carried out to substantiate the theoretical results of random walk.
\end{abstract}

\section{Keywords}

Epidemic Model, Vaccination, Continuous Time Markov Chain, Birth and Death Process, Stochastic Differential Equations

\section{Introduction}

Recently, a great interest in the analysis and prediction of consequences of public health strategies designed to control infectious disease, particularly tuberculosis and (Acquired Immune Deficiency Syndrome) AIDS [1], has arised. The epidemic model includes vaccination and is referred to as an (Susceptible-Infected-Vaccinated-Susceptible) SIVS epidemic model, where the classes contain susceptible, infective, and vaccinated individuals [1] [2]. The deterministic model was analyzed by Christopher M. Kribs-Zaleta [3]. Transmission and control of infections disease are affected by many uncertain factors, then become a stochastic process. Trend of the spread of the disease is usually only with a certain current state. That is to say, under certain conditions, each class number changes is the nature of the Mrakov process.

Birth and death process [4] is a kind of important and wide application of Markov process, the theoretical results are systematical, mature and in-depth. But various 
studies focused on the birth and death process itself, few people use it. Birth and death process in random environment have been researched by L. J. S. Allen and P. S. Mandal [5] [6].

Plentful well-known stochastic epidemic models have been used to investigate questions regarding the dynamics of an epidemic [7]-[13]. There are many studies have been investigated using stochastic models [8] [14]. Our goal in this investigation is to compare the dynamics of the deterministic and the stochastic epidemic model. The stochastic models are a continuous-time Markov chain model and a stochastic differential equation model [4] [14] [15]. The stochastic differential equation model is a new formulation that is derived from the Markov chain model.

In this paper, the continuous time Markov chain model and the stochastic differential equation model based on birth and death process [4] [14] [16] are formulated based on the deterministic epidemic model. We use the cumulative generating function to express the moment equation of the numerical characteristics of random variables and Itô stochastic differential equation [4] [14] [16] of continuous time and discrete state. In addition, at the disease-free equilibrium, it is shown that the expected values of the random variables agree with the solution to the deterministic model. Finally, through extensive numerical simulations, the comparison of deterministic model and stochastic model is given.

\section{Deterministic Epidemic Model}

Consider a model for an (Susceptible-Infected-Susceptible)SIS disease where a vaccination program is in effect, which was analyzed by C. M et al. [3]. The model consists of three differential equations, one for each of the three disease states: susceptible, infective, and vaccinated, with the number in each class denoted by $S(t), I(t)$, and $V(t)$, respectively. The system of differential equations for the deterministic epidemic model is

$$
\left\{\begin{array}{l}
\frac{\mathrm{d} S}{\mathrm{~d} t}=\mu N-\frac{\beta S I}{N}-(\mu+\phi) S+c I+\theta V, \\
\frac{\mathrm{d} I}{\mathrm{~d} t}=\frac{\beta S I}{N}+\frac{\sigma \beta V I}{N}-(\mu+c) I, \\
\frac{\mathrm{d} V}{\mathrm{~d} t}=\phi S-\frac{\sigma \beta V I}{N}-(\mu+\theta) V,
\end{array}\right.
$$

where $N$ is the constant total population size; thus we can reduce the size of the model by letting $S(t)=N-I(t)-V(t)$ and get the new model as

$$
\left\{\begin{array}{l}
\frac{\mathrm{d} I}{\mathrm{~d} t}=\beta(N-I-(1-\sigma) V) \frac{I}{N}-(\mu+c) I, \\
\frac{\mathrm{d} V}{\mathrm{~d} t}=\phi(N-I-V)-\frac{\sigma \beta V I}{N}-(\mu+\theta) V,
\end{array}\right.
$$

note that the parameters are all non-negative, and $\beta$ is the transmission rate, $\phi$ is the vaccination rate, $\mu$ is the natural death or birth rate, $\mathrm{c}$ is the recovery rate, $\theta$ is the rate of vaccine waning. The vaccine is assumed to be useful but imperfect. Thus the 
vaccine efficacy denoted by $1-\sigma, \sigma$ measures the efficiency of the vaccine as a multiplier to the infection rate: when $\sigma=0$, vaccination is hundred percent effective; and when $\sigma=1$, the vaccine is totally useless.

Although model (1) can well describe the development of the disease, in the spread and control of disease there still exist some uncertain factors such as temperature, environment of the hospital symptomtesting, etc. And the effect produced by these factors is particularly important. Based on the deterministic model (2) after dimension reduction, we take the influence of random factors on the spread of disease into considering, and establish the continuous time Markov chain (CTMC) model, namely the birth and death process.

\section{The Birth and Death Process under CTMC}

In this section, we construct a CTMC model under birth and death view for the epidemic model based on the ordinary differential equation model (2). Let $Y_{1}(t)$ and $Y_{2}(t)$ denote the discrete random variables for the number of infected and vaccinated cells at time $t$, the random variables $Y_{i}(t) \in\{0,1,2, \cdots\}$ and $t \in R_{+}, i=1,2$. Let the initial values be fixed, $Y_{1}(0)=n_{10}>0$ and $Y_{2}(0)=n_{20}>0$. The corresponding probabilities associated with the bivariate process $\left\{Y_{1}(t), Y_{2}(t)\right\}$ are as follows:

$$
p_{n_{1}, n_{2}}(t)=\operatorname{Prob}\left\{Y_{1}=n_{1}, Y_{2}(t)=n_{2} \mid Y_{1}(0)=n_{10}, Y_{2}(0)=n_{20}\right\},
$$

where $n_{i} \in\{0,1,2, \cdots\}, \quad i=1,2$. Set $\Delta Y_{i}(t) \equiv Y_{i}(t+\Delta t)-Y_{i}(t) \quad$ and $\Delta t$ be sufficiently small such that $\Delta Y_{i} \in\{-1,0,1\}$ for $i=1,2$. We formulate the continuous time Markov chain model as a birth and death process in each of the variables [4]. When time is sufficiently small, there is

$$
\begin{aligned}
& \operatorname{Prob}\left\{\Delta Y_{1}=i, \Delta Y_{2}=j \mid Y_{1}=n_{1}, Y_{2}=n_{2}\right\} \\
& = \begin{cases}b_{1} \Delta t+o(\Delta t) & (i, j)=(1,0), \\
b_{2} \Delta t+o(\Delta t) & (i, j)=(0,1), \\
d_{1} \Delta t+o(\Delta t) & (i, j)=(-1,0), \\
d_{2} \Delta t+o(\Delta t) & (i, j)=(0,-1), \\
1-\left(b_{1}+b_{2}+d_{1}+d_{2}\right) \Delta t+o(\Delta t) & (i, j)=(0,0),\end{cases}
\end{aligned}
$$

where $b_{1}=\beta n_{1}+\sigma \beta \frac{n_{1} n_{2}}{N}, b_{2}=\phi N, d_{1}=\frac{\beta}{N} n_{1}^{2}+\frac{\beta}{N} n_{1} n_{2}+(\mu+c) n_{1}$,

$d_{2}=\phi n_{1}+\phi n_{2}+\sigma \beta \frac{n_{1} n_{2}}{N}+(\mu+\theta) n_{2}$. The probabilities $p_{n_{1}, n_{2}}$ satisfy the forward

Kolmogorov differential equation [4]. Then, we have

$$
\begin{aligned}
\frac{\mathrm{d} p_{n_{1}, n_{2}}}{\mathrm{~d} t}= & {\left[\beta\left(n_{1}-1\right)+\frac{\sigma \beta}{N}\left(n_{1}-1\right) n_{2}\right] p_{n_{1}, n_{2}}(t)+\phi N p_{n_{1}, n_{2}-1}(t) } \\
& +\left[\frac{\beta}{N}\left(n_{1}+1\right)^{2}+\frac{\beta}{N}\left(n_{1}+1\right) n_{2}+(\mu+c)\left(n_{1}+1\right)\right] p_{n_{1}+1, n_{2}}(t) \\
& +\left[\phi n_{1}+\phi\left(n_{2}+1\right)+\frac{\sigma \phi}{N} n_{1}\left(n_{2}+1\right)+(\mu+\theta)\left(n_{2}+1\right)\right] p_{n_{1}, n_{2}+1}(t) \\
& -\left[\beta n_{1}+\frac{\sigma \beta}{N} n_{1} n_{2}+\phi N+\frac{\beta}{N} n_{1}^{2}+\frac{\beta}{N} n_{1} n_{2}+(\mu+c) n_{1}+\phi n_{1}+\phi n_{2}+\frac{\sigma \beta}{N} n_{1} n_{2}+(\mu+\theta) n_{2}\right] p_{n_{1}, n_{2}}(t),
\end{aligned}
$$


where $n_{1}, n_{2}=1,2, \cdots$.

In addition, when $n_{2}=0$, we have

$$
\begin{aligned}
\frac{\mathrm{d} p_{n_{1}, 0}}{\mathrm{~d} t}= & \beta\left(n_{1}-1\right) p_{n_{1}-1,0}(t)+\left[\frac{\beta}{N}\left(n_{1}+1\right)^{2}+(\mu+c)\left(n_{1}+1\right)\right] p_{n_{1}+1,0}(t) \\
& +\left[\phi n_{1}+\phi+\frac{\sigma \phi}{N} n_{1}+\mu+\theta\right] p_{n_{1}, 1}(t)-\left[\beta n_{1}+\frac{\beta}{N} n_{1}^{2}+(\mu+c) n_{1}+\phi N+\phi n_{1}\right] p_{n_{1}, 0}(t),
\end{aligned}
$$

where $n_{1}=1,2, \cdots$.

When $n_{1}=0$, we have

$$
\begin{aligned}
\frac{\mathrm{d} p_{0, n_{2}}}{\mathrm{~d} t}= & \phi N p_{0, n_{2}-1}(t)+\left[\frac{\beta}{N}+\frac{\beta}{N} n_{2}+(\mu+c)\right] p_{1, n_{2}}(t) \\
& +\left[\phi\left(n_{2}+1\right)+(\mu+\theta)\left(n_{2}+1\right)\right] p_{0, n_{2}+1}(t) \\
& -\left[\phi N+\phi n_{2}+(\mu+\theta) n_{2}\right] p_{0, n_{2}}(t),
\end{aligned}
$$

where $n_{2}=1,2, \cdots$. For $n_{1}=n_{2}=0$, there is

$$
\frac{\mathrm{d} p_{0,0}}{\mathrm{~d} t}=\left[\frac{\beta}{N}+(\mu+c)\right] p_{1,0}(t)+[\phi+(\mu+\theta)] p_{0,1}(t)-\phi N p_{0,0}(t) \text {. }
$$

The moment of the distribution corresponding to the bivariate process can be derived directly from the preceding forward Kolmogorov differential equation. The form of the moment generating function is

$$
M\left(z_{1}, z_{2}, z_{3}\right)=\sum_{n_{1}=0}^{\infty} \sum_{n_{2}=0}^{\infty} p_{n_{1}, n_{2}}(t) \mathrm{e}^{n_{1} z_{1}} \mathrm{e}^{n_{2} z_{2}}
$$

for some $z_{1}, z_{2} \in R$, the moment generating function is a solution of the partial differential equation by follows [4] [17] [18]:

$$
\begin{aligned}
\frac{\partial M\left(z_{1}, z_{2}, t\right)}{\partial t}= & \beta\left(\mathrm{e}^{z_{1}}-1\right) \frac{\partial M}{\partial z_{1}}+\frac{\sigma \beta}{N}\left(\mathrm{e}^{z_{1}}-1\right) \frac{\partial^{2} M}{\partial z_{1} \partial z_{2}}+\phi N\left(\mathrm{e}^{z_{2}}-1\right) M+\frac{\beta}{N}\left(\mathrm{e}^{-z_{1}}-1\right) \frac{\partial^{2} M}{\partial z_{1}^{2}} \\
& +\frac{\beta}{N}\left(\mathrm{e}^{-z_{1}}-1\right) \frac{\partial^{2} M}{\partial z_{1} \partial z_{2}}+(\mu+c)\left(\mathrm{e}^{-z_{1}}-1\right) \frac{\partial M}{\partial z_{1}}+\phi\left(\mathrm{e}^{-z_{2}}-1\right) \frac{\partial M}{\partial z_{1}} \\
& +\phi\left(\mathrm{e}^{-z_{2}}-1\right) \frac{\partial M}{\partial z_{2}}+\frac{\sigma \beta}{N}\left(\mathrm{e}^{-z_{2}}-1\right) \frac{\partial^{2} M}{\partial z_{1} \partial z_{2}}+(\mu+\theta)\left(\mathrm{e}^{-z_{2}}-1\right) \frac{\partial M}{\partial z_{2}} .
\end{aligned}
$$

By applying the product rule for differentiation, and differentiating both sides of the preceding differential Equation (10) with respect to $Z_{1}$, we get

$$
\begin{aligned}
\frac{\partial^{2} M\left(z_{1}, z_{2}, t\right)}{\partial z_{1} \partial t}= & \beta\left[\mathrm{e}^{z_{1}} \frac{\partial M}{\partial z_{1}}+\left(\mathrm{e}^{z_{1}}-1\right) \frac{\partial^{2} M}{\partial z_{1}^{2}}\right]+\frac{\sigma \beta}{N}\left[\mathrm{e}^{z_{1}} \frac{\partial^{2} M}{\partial z_{1} \partial z_{2}}+\left(\mathrm{e}^{z_{1}}-1\right) \frac{\partial^{3} M}{\partial z_{1}^{2} \partial z_{2}}\right]+\phi N\left(\mathrm{e}^{z_{2}}-1\right) \frac{\partial M}{\partial z_{1}} \\
& +\frac{\beta}{N}\left[-\mathrm{e}^{-z_{1}} \frac{\partial^{2} M}{\partial z_{1}^{2}}+\left(\mathrm{e}^{-z_{1}}-1\right) \frac{\partial^{3} M}{\partial z_{1}^{3}}\right]+\frac{\beta}{N}\left[-\mathrm{e}^{-z_{1}} \frac{\partial^{2} M}{\partial z_{1} \partial z_{2}}+\left(\mathrm{e}^{-z_{1}}-1\right) \frac{\partial^{3} M}{\partial z_{1}^{2} \partial z_{2}}\right] \\
& +(\mu+c)\left[-\mathrm{e}^{-z_{1}} \frac{\partial M}{\partial z_{1}}+\left(\mathrm{e}^{-z_{1}}-1\right) \frac{\partial^{2} M}{\partial z_{1}^{2}}\right]+\phi\left(\mathrm{e}^{-z_{2}}-1\right) \frac{\partial^{2} M}{\partial z_{2}^{2}}+\phi\left(\mathrm{e}^{-z_{2}}-1\right) \frac{\partial^{2} M}{\partial z_{2} \partial z_{2}} \\
& +\frac{\sigma \beta}{N}\left(\mathrm{e}^{-z_{2}}-1\right) \frac{\partial^{3} M}{\partial z_{1}^{2} \partial z_{2}}+(\mu+\theta)\left[\left(\mathrm{e}^{-z_{2}}-1\right) \frac{\partial^{2} M}{\partial z_{1} \partial z_{2}}\right] .
\end{aligned}
$$


Take $z_{1}=0=z_{2}$ in Equation (10), the equations for expectation of $Y_{1}$ is:

$$
\frac{\mathrm{d} E\left[Y_{1}(t)\right]}{\mathrm{d} t}=\beta E\left[Y_{1}\right]+\frac{\sigma \beta}{N} E\left[Y_{1} Y_{2}\right]-\frac{\beta}{N} E\left[Y_{1}^{2}\right]-\frac{\beta}{N} E\left[Y_{1} Y_{2}\right]-(\mu+c) E\left[Y_{1}\right] .
$$

Differentiating both sides of the preceding differential Equation (10) with respect to $z_{2}$, it shows that

$$
\begin{aligned}
\frac{\partial^{2} M\left(z_{1}, z_{2}, t\right)}{\partial z_{2} \partial t}= & \beta\left(\mathrm{e}^{z_{1}}-1\right) \frac{\partial^{2} M}{\partial z_{1} \partial z_{2}}+\frac{\sigma \beta}{N}\left(\mathrm{e}^{z_{1}}-1\right) \frac{\partial^{3} M}{\partial z_{1} \partial z_{2}^{2}}+\phi N\left[\mathrm{e}^{z_{2}} M+\left(\mathrm{e}^{z_{2}}-1\right) \frac{\partial M}{\partial z_{2}}\right] \\
& +\frac{\beta}{N}\left(\mathrm{e}^{-z_{1}}-1\right) \frac{\partial^{3} M}{\partial z_{1}^{2} z_{2}}+\frac{\beta}{N}\left(\mathrm{e}^{-z_{1}}-1\right) \frac{\partial^{3} M}{\partial z_{1} z_{2}^{2}}+(\mu+c)\left(\mathrm{e}^{-z_{1}}-1\right) \frac{\partial^{2} M}{\partial z_{1} \partial z_{2}} \\
& +\phi\left[-\mathrm{e}^{-z_{2}} \frac{\partial M}{\partial z_{1}}+\left(\mathrm{e}^{-z_{2}}-1\right) \frac{\partial^{2} M}{\partial z_{1} \partial z_{2}}\right]+\phi\left[-\mathrm{e}^{-z_{2}} \frac{\partial M}{\partial z_{2}}+\left(\mathrm{e}^{-z_{2}}-1\right) \frac{\partial^{2} M}{\partial z_{2}^{2}}\right] \\
& +\frac{\sigma \beta}{N}\left[-\mathrm{e}^{-z_{2}} \frac{\partial^{2} M}{\partial z_{1} \partial z_{2}}+\left(\mathrm{e}^{-z_{2}}-1\right) \frac{\partial^{3} M}{\partial z_{1} \partial z_{2}^{2}}\right]+(\mu+\theta)\left[-\mathrm{e}^{-z_{2}} \frac{\partial M}{\partial z_{2}}+\left(\mathrm{e}^{-z_{2}}-1\right) \frac{\partial^{2} M}{\partial z_{2}^{2}}\right] .
\end{aligned}
$$

Equation (10) then gives the following differential equations for the expectation of $Y_{2}$ by substituting $z_{1}=0=z_{2}$ :

$$
\frac{\mathrm{d} E\left[Y_{2}(t)\right]}{\mathrm{d} t}=\phi N-\phi E\left[Y_{1}\right]-\phi E\left[Y_{2}\right]-\frac{\sigma \beta}{N} E\left[Y_{1} Y_{2}\right]-(\mu+\theta) E\left[Y_{2}\right]
$$

From all of the above, we get

$$
\begin{aligned}
\frac{\mathrm{d} E\left[Y_{1}(t)\right]}{\mathrm{d} t}+\frac{\mathrm{d} E\left[Y_{2}(t)\right]}{\mathrm{d} t}= & \beta E\left[Y_{1}\right]+\frac{\sigma \beta}{N} E\left[Y_{1} Y_{2}\right]-\frac{\beta}{N} E\left[Y_{1}^{2}\right]-\frac{\beta}{N} E\left[Y_{1} Y_{2}\right]-(\mu+c) E\left[Y_{1}\right] \\
& +\phi N-\phi E\left[Y_{1}\right]-\phi E\left[Y_{2}\right]-\frac{\sigma \beta}{N} E\left[Y_{1} Y_{2}\right]-(\mu+\theta) E\left[Y_{2}\right] .
\end{aligned}
$$

To enlarge type on the right side, it follows that

$$
\frac{\mathrm{d}\left(E\left[Y_{1}\right]+E\left[Y_{2}\right]\right)}{\mathrm{d} t} \leq \phi N-(\phi+\mu+c-\beta) Y_{1}-(\phi+\mu+\theta) Y_{2} .
$$

Take

$$
\hat{\phi}=\min \{\phi+\mu+c-\beta, \phi+\mu+\theta\}
$$

then

$$
\frac{\mathrm{d}\left(E\left[Y_{1}\right]+E\left[Y_{2}\right]\right)}{\mathrm{d} t} \leq \phi N-\hat{\phi}\left(E\left[Y_{1}\right]+E\left[Y_{2}\right]\right)
$$

This represents that $Y_{1}$ and $Y_{2}$ are constrained by certain conditions, so the expectations of the random variables lie in the region

$$
0 \leq \frac{\mathrm{d}\left(E\left[Y_{1}\right]+E\left[Y_{2}\right]\right)}{\mathrm{d} t} \leq \frac{\phi N}{\hat{\phi}} .
$$

For the above $\hat{\phi}$, the number of infected and vaccinated also lies in this region. And the set

$$
0 \leq I+V \leq \frac{\phi N}{\hat{\phi}}
$$


is invariant. Therefore, the expectations have the same property as the variables in the deterministic model.

\section{Itô Stochastic Differential Equations}

Based on the CTMC model, Itô SDEs can be derived by applying the methods in [17] [19]. The possible changes of CTMC model are given in Table 1, similar to the Markov chain model.

Let $X_{1}(t)$ and $X_{2}(t)$ denote continuous random variables for the side of $\mathrm{I}$ and $\mathrm{V}$, respectively. Then $\boldsymbol{X}(t)=\left(X_{1}(t), X_{2}(t)\right)^{\mathrm{T}}$ is a vector random variable defined on an appropriately defined sample space, where

$$
X_{1}, X_{2} \in[0, \infty), t \in[0, \infty) .
$$

As in the CTMC model, it is assumed that the time interval $\Delta t$ is sufficiently small, so that at most one birth or death occur in this time interval. Let

$\Delta \boldsymbol{X}=\Delta \boldsymbol{X}(t)=\left(\Delta X_{1}(t), X_{2}(t)\right)$, where $\Delta X_{i}(t)=X_{i}(t+\Delta t)-X_{i}(t), i=1,2$. The ith change is denoted by $(\Delta \boldsymbol{X})_{i}$. Terms $o(\Delta t)$ are neglected. For sufficiently small $\Delta t$, the expectation vector of $\Delta \boldsymbol{X}$ is

$$
E(\Delta \boldsymbol{X}) \approx\left[(1,0)^{\mathrm{T}} b_{1}+(-1,0)^{\mathrm{T}} d_{1}\right] \Delta t+\left[(1,0)^{\mathrm{T}} b_{2}+(-1,0)^{\mathrm{T}} d_{2}\right] \Delta t .
$$

In addition, the variance of $\Delta X_{i}, i=1,2$ is defined as $\sigma_{i}^{2}=\sigma_{i i}=E\left[\left(\Delta X_{i}\right)^{2}\right]-\left[\mu_{i} \Delta t\right]^{2}$, then the covariance of $\Delta X_{1}$ and $\Delta X_{2}$ is $\sigma_{12}=E\left(\Delta X_{1} \Delta X_{2}\right)-\mu_{1} \mu_{2}(\Delta t)^{2}$. The covariance matrix for $\Delta \boldsymbol{X}$ is

$$
\Sigma \Delta t=E[\Delta \boldsymbol{X}]\left(E[\Delta \boldsymbol{X}]^{\mathrm{T}}\right)-E[\Delta \boldsymbol{X}](\Delta \boldsymbol{X})^{\mathrm{T}} \approx E\left[\Delta \boldsymbol{X}(\Delta \boldsymbol{X})^{\mathrm{T}}\right],
$$

where $E[\Delta \boldsymbol{X}]\left(E[\Delta \boldsymbol{X}]^{\mathrm{T}}\right)$ is neglected since it is $o(\Delta t)^{2}$.

$$
\Sigma \Delta t=E\left(\begin{array}{cc}
\left(\Delta X_{1}\right)^{2} & \Delta X_{1} \Delta X_{2} \\
\Delta X_{1} \Delta X_{2} & \left(\Delta X_{2}\right)^{2}
\end{array}\right) \approx\left(\begin{array}{ll}
\sigma_{11} & \sigma_{12} \\
\sigma_{21} & \sigma_{22}
\end{array}\right),
$$

hence

$$
\sigma_{i i}=E\left[\left(\Delta X_{i}\right)^{2}\right] \approx\left[(1)^{2} b_{i}+(-1)^{2} d_{i}\right] \Delta t
$$

Table 1. Possible changes in $\Delta \boldsymbol{X}$.

\begin{tabular}{ccc}
\hline$i$ & $(\Delta \boldsymbol{x})_{i}^{T}$ & $p_{i} \Delta t$ (The probability) \\
\hline 1 & $(1,0)$ & $p_{1} \Delta t=b_{1} \Delta t=\left(\beta X_{1}+\frac{\sigma \beta}{N} X_{1} X_{2}\right) \Delta t$ \\
2 & $(0,1)$ & $p_{2} \Delta t=b_{2} \Delta t=(\phi N) \Delta t$ \\
3 & $(-1,0)$ & $p_{3} \Delta t=d_{1} \Delta t=\left(\frac{\beta}{N} X_{1}^{2}+\frac{\beta}{N} X_{1} X_{2}+(\mu+c) X_{1}\right) \Delta t$ \\
4 & $(0,-1)$ & $p_{4} \Delta t=d_{2} \Delta t=\left(\phi X_{1}+\phi X_{2}+\frac{\sigma \beta}{N} X_{1} X_{2}+(\mu+\theta) X_{2}\right) \Delta t$ \\
5 & $(0,0)$ & $p_{5} \Delta t=1-\sum_{i=1}^{4} p_{i} \Delta t=1-\left(b_{1}+b_{2}+d_{1}+d_{2}\right) \Delta t$ \\
\hline
\end{tabular}


where $i=1,2$. In addition, we also have

$$
\sigma_{i j}=E\left[\left(\Delta X_{i}\right)\left(\Delta X_{j}\right)\right] \approx 0,
$$

for $i \neq j$. Therefore, the covariance matrix $\Sigma$ can be approximated as follows [19]:

$$
\Sigma=\left(\begin{array}{cc}
b_{1}+d_{1} & 0 \\
0 & b_{2}+d_{2}
\end{array}\right)=B^{2} .
$$

For sufficiently small $\Delta t$ and large $\boldsymbol{X}(t), \Delta \boldsymbol{X}(t)$ has an approximate normal distribution with mean $\mu \Delta t$ and covariance matrix $B^{2} \Delta t$, where $B=\left(B_{i j}\right)$, see [15] [19]. Let $\boldsymbol{\eta}=\left(\eta_{1}, \eta_{2}\right)^{\mathrm{T}} \sim N(0, I)$. Then there is $\boldsymbol{\mu} \Delta t+B(\sqrt{\Delta t} \boldsymbol{\eta}) \sim N\left(\boldsymbol{\mu}, B^{2} \Delta t\right)$ and the approximation to $\boldsymbol{X}(t+\Delta t)$ :

$$
\boldsymbol{X}(t+\Delta t)=\boldsymbol{X}(t)+\Delta \boldsymbol{X}(t)=\boldsymbol{X}(t)+\boldsymbol{\mu} \Delta t+B(\sqrt{\Delta t}) \boldsymbol{\eta}
$$

Due to that the preceding expression is an Euler-Maruyama approximation to a system of Itô stochastic differential equation [20], that is to say, the system (28) can converge in the mean square sense to the system of Itô stochastic differential equation as follows:

$$
\mathrm{d} \boldsymbol{X}=\boldsymbol{\mu} \mathrm{d} t+B \mathrm{~d} \boldsymbol{W},
$$

where $\boldsymbol{\mu}$ and $B$ mean the drift term and the diffusion matrix, respectively. Thereby the system can be represented by the following formula:

$$
\left\{\begin{aligned}
\mathrm{d} X_{1}= & \left(b_{1}-d_{1}\right) \mathrm{d} t+\sqrt{\left(b_{1}+d_{1}\right)} \mathrm{d} W_{1}(t) \\
= & \left(\beta X_{1}+\frac{\sigma \beta}{N} X_{1} X_{2}-\frac{\beta}{N} X_{1}^{2}-\frac{\beta}{N} X_{1} X_{2}-(\mu+c) X_{1}\right) \mathrm{d} t \\
& +\sqrt{\beta X_{1}+\frac{\sigma \beta}{N} X_{1} X_{2}+\frac{\beta}{N} X_{1}^{2}+\frac{\beta}{N} X_{1} X_{2}+(\mu+c) X_{1}} \mathrm{~d} W_{1}(t), \\
\mathrm{d} X_{2}= & \left(b_{2}-d_{2}\right) \mathrm{d} t+\sqrt{\left(b_{2}+d_{2}\right)} \mathrm{d} W_{2}(t) \\
= & \left(\phi N-\phi X_{1}-\phi X_{2}-\frac{\sigma \beta}{N} X_{1} X_{2}-(\mu+c) X_{2}\right) \mathrm{d} t \\
& +\sqrt{\phi N+\phi X_{1}+\phi X_{2}+\frac{\sigma \beta}{N} X_{1} X_{2}+(\mu+c) X_{2}} \mathrm{~d} W_{2}(t),
\end{aligned}\right.
$$

where $W_{1}(t)$ and $W_{2}(t)$ are two independent Wiener processes.

Note 1: The form of the Itô stochastic differential equation is not unique. From the reference [18], the form of Equation (30) can be expressed in other ways as the equivalent stochastic differential equations with the same joint probability density like

$$
\mathrm{d} \boldsymbol{X}=\boldsymbol{\mu} \mathrm{d} t+T \mathrm{~d} \boldsymbol{W}^{*},
$$

where $T T^{\mathrm{T}}=B^{2}=\Sigma$ and

$$
T=\left(\begin{array}{cccc}
\sqrt{b_{1}} & \sqrt{d_{1}} & 0 & 0 \\
0 & 0 & \sqrt{b_{2}} & \sqrt{d_{2}}
\end{array}\right)
$$

with $W^{*}=\left(W_{1}^{*}, \cdots, W_{4}^{*}\right)^{\mathrm{T}}$ a vector of four independent Wiener process [4] [19] and it represent independent standard Brownian motion respectively. 
Note 2: Birth and death rates can be varied in forms. In the preceding formulations, we have assumed that the per capita birth and death rates of the population are positive and negative, respectively. In fact, we can take the birth and death rates from the Itô stochastic differential equation.

For example, let

$$
\begin{array}{ll}
b_{1}(I, V)=a_{11} I+a_{12} V, & d_{1}(I, V)=b_{11} I+b_{12} V+\frac{\beta}{N} I^{2}, \\
b_{2}(I, V)=a_{21} I+a_{22} V, & d_{2}(I, V)=b_{21}+b_{22} V+\phi I+\frac{\beta}{N} I V,
\end{array}
$$

where $a_{i j}$ and $b_{i j}$ are positive constants, $i, j=1,2$, and satisfy the following relations:

$$
\begin{aligned}
& a_{i 1}-b_{i 1}=\alpha_{i}, \quad \alpha_{i 2}-b_{i 2}=\beta_{i}, \\
& \alpha_{i}=\left(\alpha_{1}, \alpha_{2}\right)^{\mathrm{T}}=(\beta-(\mu+c), \phi N)^{\mathrm{T}}, \beta_{i}=\left(\beta_{1}, \beta_{2}\right)^{\mathrm{T}}=\left(\frac{\sigma \beta-\beta}{N},-(\phi \mu+\theta)\right)^{\mathrm{T}},
\end{aligned}
$$

for redefined $b_{i}$ and $d_{i}$, we can get the following Itô stochastic differential equation by a similar method.

$$
\left\{\begin{aligned}
\mathrm{d} X_{1}= & \left(b_{1}-d_{1}\right) \mathrm{d} t+\sqrt{\left(b_{1}+d_{1}\right)} \mathrm{d} W_{1}(t) \\
= & \left(\beta X_{1}+\frac{\sigma \beta}{N} X_{1} X_{2}-\frac{\beta}{N} X_{1}^{2}-\frac{\beta}{N} X_{1} X_{2}-(\mu+c) X_{1}\right) \mathrm{d} t \\
& +\sqrt{\beta X_{1}+\frac{\sigma \beta}{N} X_{1} X_{2}+\frac{\beta}{N} X_{1}^{2}+\frac{\beta}{N} X_{1} X_{2}+(\mu+c) X_{1}} \mathrm{~d} W_{1}(t), \\
\mathrm{d} X_{2}= & \left(b_{2}-d_{2}\right) \mathrm{d} t+\sqrt{\left(b_{2}+d_{2}\right)} \mathrm{d} W_{2}(t) \\
= & \left(\phi N-\phi X_{1}-\phi X_{2}-\frac{\sigma \beta}{N} X_{1} X_{2}-(\mu+c) X_{2}\right) \mathrm{d} t \\
& +\sqrt{\phi N+\phi X_{1}+\phi X_{2}+\frac{\sigma \beta}{N} X_{1} X_{2}+(\mu+c) X_{2}} \mathrm{~d} W_{2}(t),
\end{aligned}\right.
$$

it is clear that the terms in the Wiener processes of the Itô stochastic differential equation are greater in model (35) since $a_{i 1}+b_{i 1}>\alpha_{i}, a_{i 2}+b_{i 2}>\beta_{i}$ for $i=1,2$.

\section{Numerical Simulation}

Numerical simulations are used to illustrate the dynamics of the deterministic model, continuous time Markov chain model and stochastic differential equation model. We simulate the birth and death process perspective of infectious disease model by applying MATLAB. Throughout the paper, we choose the parameters as: $\mu=0.01$, $c=0.01, \theta=0.01, \sigma=0.02, \beta=0.27, \phi=0.1, N=500$. Take initial values $\left(n_{10}, n_{20}\right)=(300,150)$. The stable equilibrium in the deterministic model is $E^{*}=\left(I^{*}, V^{*}\right)=(320.4,145.4718)$ which is a global attractor.

Figure 1(a) and Figure 1(b) respectively display two sample paths of the stochastic model graphed with the ordinary differential equation. It can be clearly seen from the dashed line in Figure 1 that with the change of time, infected number are gradually 


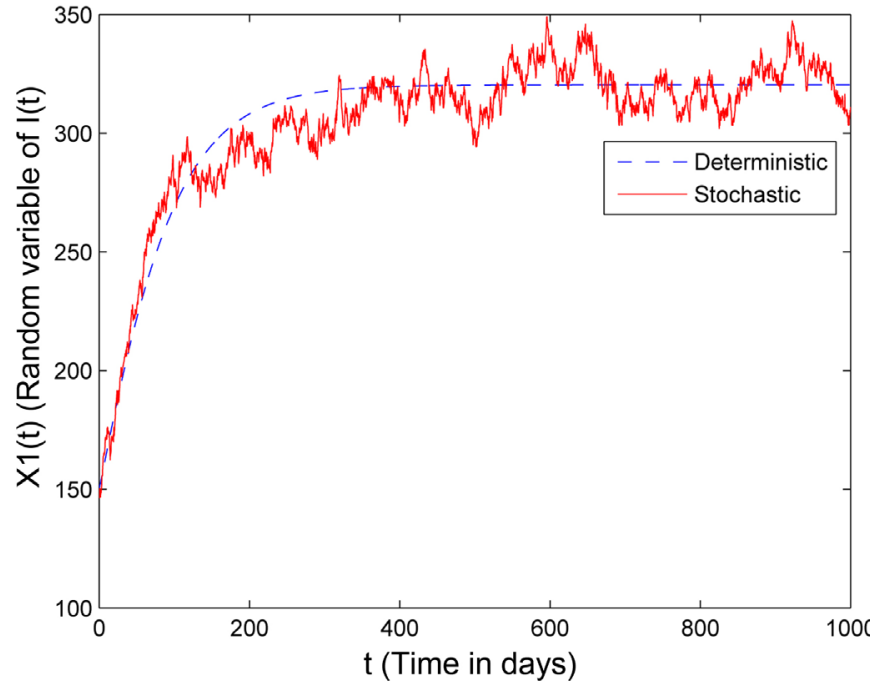

(a)

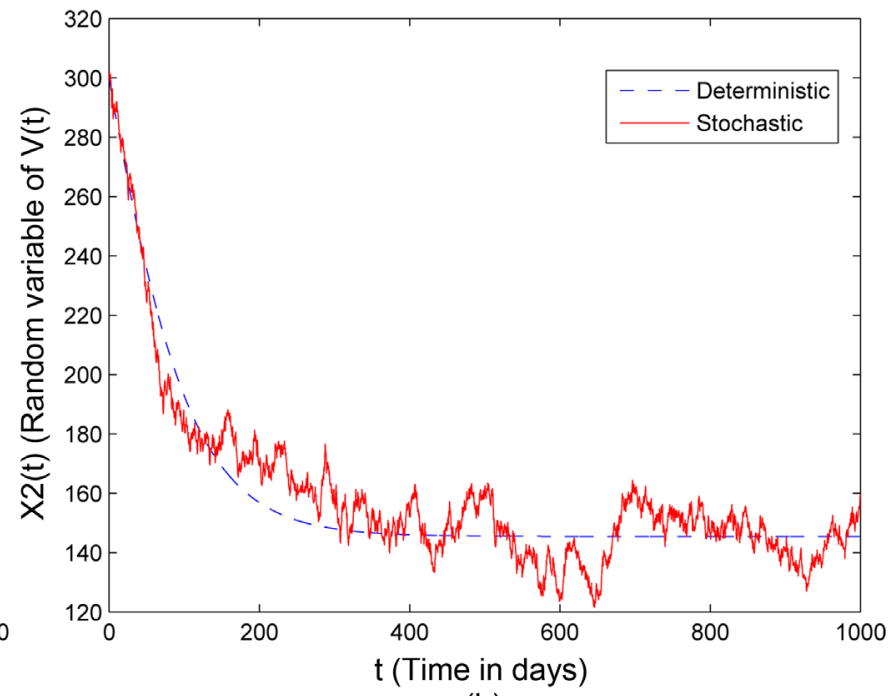

(b)

Figure 1. The solution curves of deterministic model and stochastic model. In (a) and (b), the ordinary differential equation solution is attracted to the equilibrium $E^{*}=\left(I^{*}, V^{*}\right)=(320.4,145.4718)$ with initial conditions $\left(n_{10}, n_{20}\right)=(300,150)$. Parameter values are given as follows: $\mu=0.01, c=0.01, \theta=0.01, \sigma=0.02, \beta=0.27, \phi=0.1, N=500$.

increased and close to the equilibrium value. At the same time, vaccinated number are gradually reduced. One sample path for the stochastic differential equation models (30) and (35) is graphed in Figure 1(a) and Figure 1(b) with the solid line. Figure 1 shows the solution of the stochastic model fluctuates around the solution of deterministic model due to weak noise intensities which reflects that the disease will persistent.

At $t=1000,15,000$ sample paths are used to compute probability histograms for the stochastic equation models (30) and (35). The initial conditions lie in the basin of attraction for the stable endemic equilibrium for the deterministic model is $\left(n_{10}, n_{20}\right)=(300,150)$. The two models of the probability distributions are visible in Figure 2(a) and Figure 2(b). The graphs in Figure 2(a) and Figure 2(b) are computed from the CTMC model.

The number of infected people is represented by the horizontal axis and vaccinated people is represented by the vertical axis. A two-dimensional random walk is given in Figure 3. It's easy to see the path of the random walk around the equilibrium point $E^{*}=\left(I^{*}, V^{*}\right)=(320.4,145.4718)$.

\section{Conclusions}

In this paper, two new stochastic epidemic models, namely, a continuous time Markov model and a stochastic differential epidemic model, are formulated to account for the variability inherent in the birth, death, and infection process. Our goals are to provide the solution of the stochastic model and CTMC model fluctuates around the endemic disease equilibrium $E^{*}$, and the average fluctuations around the endemic disease equilibrium $E^{*}$ in time are small due to the weak noise intensities. Furthermore, we get the path of the random walk of infected number and vaccinated number also 


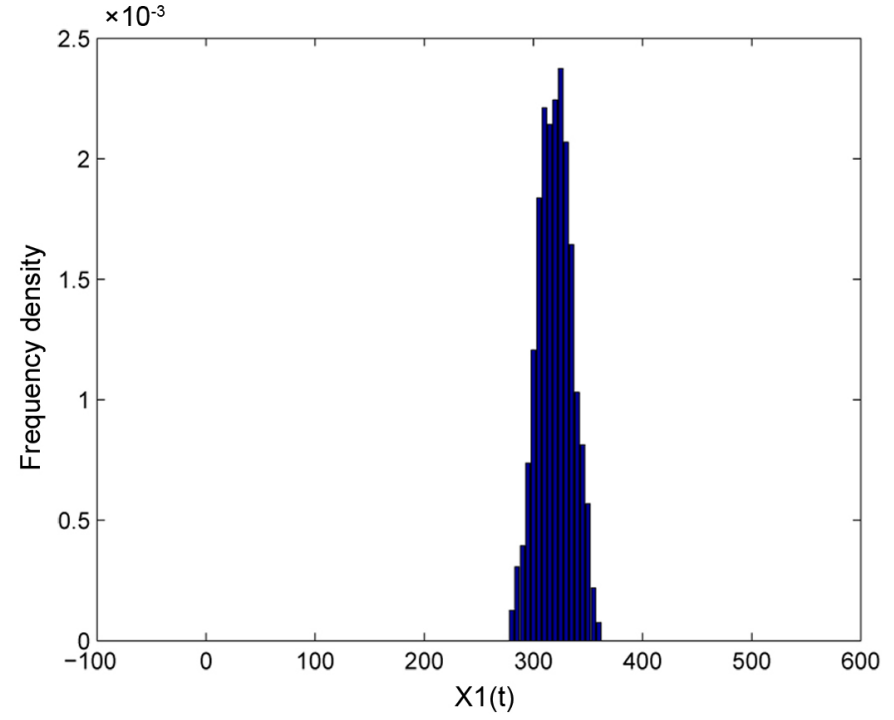

(a)

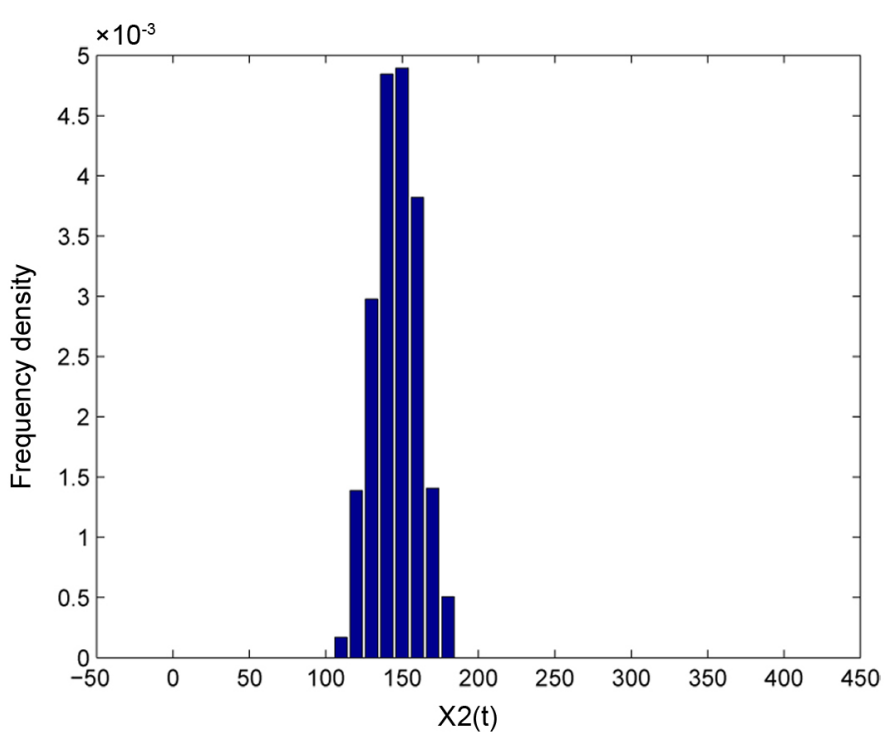

(b)

Figure 2. Probability histograms for infected number and vaccinated number distribution for taken 1000 sample path. The parameter values are $\mu=0.01, c=0.01, \theta=0.01, \sigma=0.02, \beta=0.27, \phi=0.1, N=500$, and the initial condition is $\left(n_{10}, n_{20}\right)=(300,150)$.

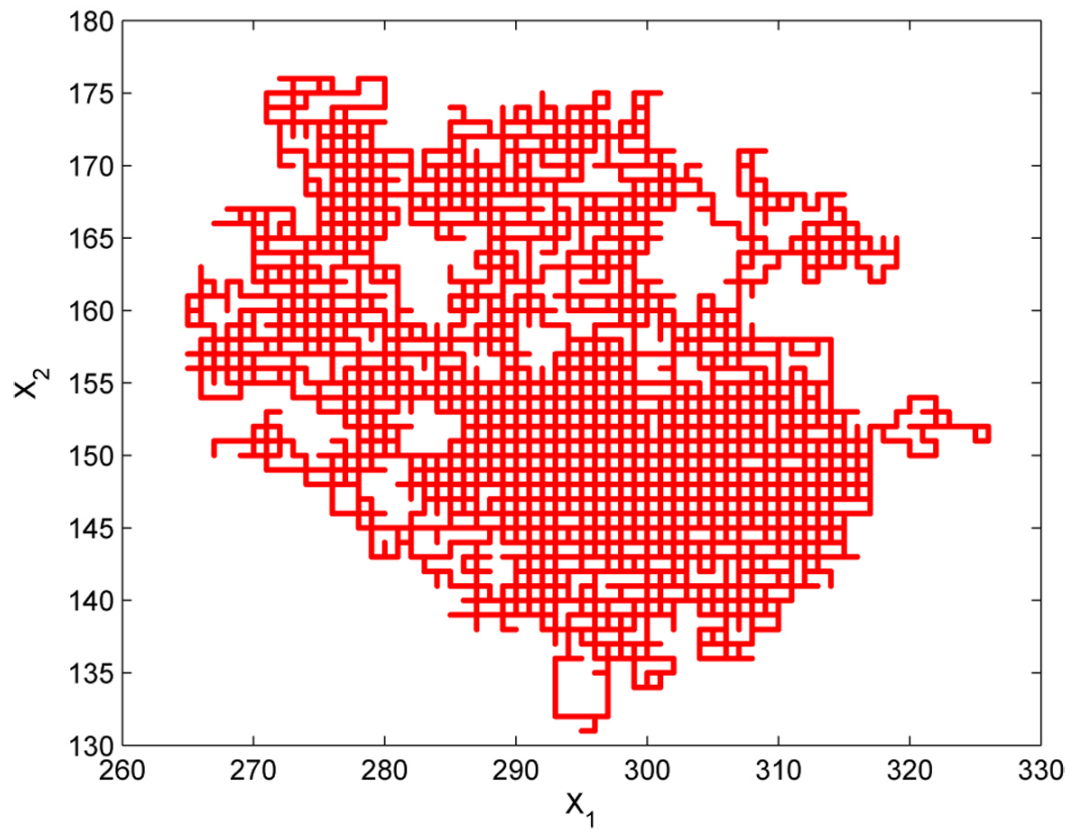

Figure 3. Random walk picture of infected number and vaccinated number in Markov model. Parameter values are $\mu=0.01, c=0.01, \theta=0.01, \sigma=0.02$, $\beta=0.27, \phi=0.1, N=500$, and the initial condition is $\left(n_{10}, n_{20}\right)=(300,150)$.

around the equilbrium point.

The continuous time Markov chain model is preferred over the stochastic differential equation model because the continuous time Markov chain model preserves the discrete population values. We also derived the formula for the fluctuation of the 
solution around the endemic equilibrium and obtained the ergodicity of the stochastic model. Computer simulations are presented to verify our theoretical results. Based on the parameter value, simulations of the CTMC model show for a population size $\mathrm{N}=$ 500 and depending on the initial values, introduction of a small number of infective individuals into a population can have similar long term outcomes in the stochastic model. We found that weak noise intensities affect long term behavior of each state slightly. These results regarding population size and choice of deterministic versus stochastic model apply to the pertussis model but may hold for more general epidemic models when the population is homogeneously mixed.

\section{Acknowledgements}

We are grateful to the editor and referees for their valuable comments that greatly improved the presentation of this paper.

\section{References}

[1] Rao, P.R.S. and Kumar, M.N. (2015) A Dynamic Model for Infectious Diseases: The Role of Vaccination and Treatment. Chaos, 75, 34-49. http://dx.doi.org/10.1016/j.chaos.2015.02.004

[2] Zhou, Y.L. and Wang, H.Q. (2010) Analysis Of global Stability for an Epidemic Model with Quarantine and Vaccination. University of Shanghai for Science and Technology, 32, 249252.

[3] Kribs-Zaleta, C.M. and Velasco-Hernandez, J.X. (2000) A Simple Vaccination Model with Multiple Endemic States. Mathematical Biosciences, 164, 183-201. http://dx.doi.org/10.1016/S0025-5564(00)00003-1

[4] Allen, L.J.S. (2010) An Introduction to Stochastic Processes with Applications to Biology. 2nd Edition, Chapman and Hall/CRC Press, Boca Raton.

[5] Allen, L.J.S. (2006) Stochastic Epidemic Models with a Backward Bifurcation. Mathermactical Biosciences and Engineering, 3, 445-458. http://dx.doi.org/10.3934/mbe.2006.3.445

[6] Mandal, P.S., Allen, L.J.S. and Banerjee, M. (2014) Stochastic Modeling of Phytoplankton Allelopathy. Applied Mathematical Modelling, 38, 1583-1596. http://dx.doi.org/10.1016/j.apm.2013.08.031

[7] Zhang, Y.H., Xu, C.Q. and Yuan, S.L. (2015) Stochastic SIS Epidemic Model with Contract Rate Influence by Noise. University of Shanghai for Science and Technology, 37, 511516.

[8] Kirupaharan, N. and Allen, L.J.S. (2004) Coexistence of Multiple Pathogen Strains in Stochastic Epidemic Models with Density-Dependent Mortality. Bulletin of Mathematical Biology, 66, 841-864. http://dx.doi.org/10.1016/j.bulm.2003.11.007

[9] Gray, A., Greenhalgh, D., Hu, 1., Mao, X. and Pan, J. (2011) A Stochastic Differential Equation Sis Epidemic Model. SIAM: SIAM Journal on Applied Mathematics, 71, 876-902. http://dx.doi.org/10.1137/10081856X

[10] Henson, S.M., King, A.A., Costantino, R.F., Cushing, J.M., Dennis, B. and Desharnais, R.A. (2003) Explaining and Predicting Patterns in Stochastic Population Systems. Proceedings of the Royal Society of London B, 270, 1549-1553.

[11] Isham, V. (1991) Assessing the Variability of Stochastic Epidemics. Mathematical Biosciences, 107, 209-224. http://dx.doi.org/10.1016/0025-5564(91)90005-4 
[12] Van Kampen, N.G. (1976) Stochastic Differential Equations. Physics Reports (Section C of Physics Letters), 24, 171-228. http://dx.doi.org/10.1016/0370-1573(76)90029-6

[13] Nasell, I. (1999) On the Quasi-Stationary Distribution of the Stochastic Logistic Epidemic. Mathematical Biosciences, 156, 21-40. http://dx.doi.org/10.1016/S0025-5564(98)10059-7

[14] Allen, L.J.S. (1945) An Introduction to Stochastic Epidemic Models. Mathematical Epidemiology. http://link.springer.com/chapter/10.1007\%2F978-3-540-78911-6 3

[15] Kurtz, T.G. (1971) Limit Theorems for Sequences of Jump Markov Process Approximating Ordinary Differential Processes. Journal of Applied Probability, 8, 344-356. http://dx.doi.org/10.1017/S002190020003535X

[16] Allen, E.J., Allen, L.J.S., Arciniega, A. and Greenwood, P.E. (2008) Construction of Equivalent Stochastic Differential Equation Models. Stochastic Analysis and Applications, 26, 274297. http://dx.doi.org/10.1080/07362990701857129

[17] Lloyd, A.L. (2004) Estimating Variability in Models for Recurrent Epidemics: Assessing the Use of Moment Closure Techniques. Theoretical Population Biology, 65, 49-65. http://dx.doi.org/10.1016/j.tpb.2003.07.002

[18] Ekanayake, A.J. and Allen, L.J.S. (2010) Comparison of Markov Chain and Stochastic Differential Equation Population Models Under Higher-Order Moment Closure Approximations. Stochastic Analysis and Applications, 28, 907-927. http://dx.doi.org/10.1080/07362990903415882

[19] Allen, E. (2007) Modeling with Itô Stochastic Differential Equations. Springer, Dordrecht.

[20] Milosevic, M. (2016) The Euler-Maruyama Approximation of Solution to Stochastic Differential Equations with Piecewise Constant Arguments. Journal of Computational and Applied Mathematics, 298, 1-12. http://dx.doi.org/10.1016/j.cam.2015.11.019

\section{Submit or recommend next manuscript to SCIRP and we will provide best service for you:}

Accepting pre-submission inquiries through Email, Facebook, LinkedIn, Twitter, etc.

A wide selection of journals (inclusive of 9 subjects, more than 200 journals)

Providing 24-hour high-quality service

User-friendly online submission system

Fair and swift peer-review system

Efficient typesetting and proofreading procedure

Display of the result of downloads and visits, as well as the number of cited articles

Maximum dissemination of your research work

Submit your manuscript at: http://papersubmission.scirp.org/

Or contact jamp@scirp.org 